\title{
Labyrinthe
}

16 | 2003

Afrique(s)

\section{Isabelle Jan, Divinités du tic. En lisant Charles Dickens}

\section{Christophe Annoussamy}

\section{(2) OpenEdition}

\section{Journals}

Édition électronique

URL : http://journals.openedition.org/labyrinthe/320

DOI : $10.4000 /$ labyrinthe.320

ISSN : 1950-6031

Éditeur

Hermann

Édition imprimée

Date de publication : 1 décembre 2003

\section{Référence électronique}

Christophe Annoussamy, «Isabelle Jan, Divinités du tic. En lisant Charles Dickens », Labyrinthe [En ligne], 16 | 2003, mis en ligne le 17 juin 2008, consulté le 22 septembre 2020. URL : http://

journals.openedition.org/labyrinthe/320 ; DOI : https://doi.org/10.4000/labyrinthe.320

Propriété intellectuelle 


\section{Isabelle Jan, Divinités du tic. En lisant Charles Dickens, Éditions du Rocher, 2003}

Christophe AnNOUSSAMY*

Spécialiste de littérature enfantine, Isabelle Jan n'entend pourtant pas réduire Dickens au poète de l'enfance, et encore moins à cette "niaiserie », comme elle la qualifie, d'un écrivain pour les enfants. Elle prétend dénoncer les clichés les plus tenaces qui s'attachent à la réputation de l'auteur, afin de le mettre « à sa vraie place », écrit-elle, « celle d'un immense artiste, maître du langage et annonciateur de frissons inconnus ». Ce n'est qu'à moitié qu'elle tient un pari dont on peut penser, par ailleurs, qu'il est lui-même devenu un lieu commun de la recherche universitaire récente sur le grand romancier anglais.

L'essai d'Isabelle Jan, dont le titre reprend en partie celui de l'ouvrage que le philosophe Alain consacrait au même auteur, constitue en de nombreux points une habile synthèse de l'œuvre de Dickens, dont il commence par envisager la dimension cinématographique, en esquissant un rapprochement avec les films de Charlie Chaplin. Les deux hommes auraient partagé une « esthétique » du « charabia et [de] la mimique », en créant, dans leurs œuvres respectives, « des êtres para-humains, réduits à une mimique obsédante et outrée, existence improbable, imposée cependant avec la force du mythe ». L'auteur s'intéresse alors plus particulièrement à un type de figure, celui de l'enfance malheureuse, omniprésente, on le sait, dans les romans de Dickens. Ce « défilé de silhouettes burlesques et pathétiques » doit-il éternellement renvoyer à l' autobiographie du romancier, comme le veut la tradition critique ? Isabelle Jan veut prouver que cette représentation tient à la fois du mythe et de la réalité. Quelques rappels biographiques suffisent à montrer que l'existence du romancier ne se réduit pas aux malheurs de ses jeunes personnages. Le projet dickensien n'est pas, en effet, « de faire revivre le passé, mais de faire vivre un héros », dans des récits qui tendent vers la forme du conte. Isabelle Jan s'étonne néanmoins qu'après Les Aventures de M. Pickwick, œuvre « ensoleillée », qu'elle qualifie de « chef-d'œuvre des chefs-d'œuvre » dickensiens, le romancier se soit penché vers des sujets plus noirs, dont certaines nouvelles insérées dans le récit de Pickwick annoncent déjà la tonalité. Au couple formé par Pickwick et son valet Sam Weller succèdent des figures d'enfants

* Christophe Annoussamy est actuellement Ater à l'université Paris IV-Sorbonne. Il prépare, sous la direction de Pierre Brunel, une thèse de littérature comparée sur Charles Dickens et le monde victorien dans l'œuvre de Julien Green (soutenance prévue fin 2004). 
martyrs, Olivier Twist, Paul Dombey, Pip, où, à chaque fois, le récit «ne demeure libre et vivant que grâce aux coups que reçoit un enfant ». Et Isabelle Jan de s'interroger: "Comment expliquer que cette œuvre qui s'est ouverte de façon aussi candide, dans la pure joie d'être au monde, change si brutalement d'orientation et se tourne du côté de l'enfer? L'aurore de Pickwick est aussitôt occultée et le deuxième roman, Olivier Twist, oublie Merry England et l'échange contre la crasse et les ténèbres ». L'essai se présente dès lors comme une enquête, qui s'efforce de remonter à la source d'un mystère.

Le troisième chapitre de l'ouvrage se penche ainsi sur la création des personnages, dont le lecteur de Dickens ne percevrait que des fragments. Le romancier les dessinerait à partir de leur squelette, comme le suggère la figure grimaçante d'Uriah Heep, dans David Copperfield. «C'est de la manière dont leurs os sont agencés », écrit Isabelle Jan, que ces personnages « prennent vie »; mais cet agencement n'a « ni cause, ni finalité ». Il dessine les grands traits d'une anatomie « délirante » mais qui, pour autant, n'est « pas toujours comique ». Isabelle Jan montre ensuite (chapitre 4) comment ces figures sont vouées à la solitude, dans un univers où la communication avec autrui se révèle impossible. Les personnages se livrent à une course vers l'abîme qui entraîne un rapport particulier avec la mort, dont traite le cinquième chapitre. L'oxymore apparent de son titre, «Hilarantes funérailles », ne doit pas tromper. Pour Isabelle Jan, en effet, la mort chez Dickens est « un accident libérateur et souvent joyeux ». Le cimetière y devient le « refuge ultime, celui des enfants abandonnés », et, par opposition, c'est « grâce à la mort inaugurale [que] la fiction est enfin autorisée et va pouvoir se déployer. Par la magie d'une seule mort, ce sont les moribonds eux-mêmes qui vont pouvoir, à présent, occuper tout l'espace [...]». Ainsi les enfants ne sont-ils jamais véritablement « reliés à leurs parents, ni définis par eux »: ils sont « directement issus des morts », comme le suggère la scène inaugurale des Grandes Espérances. Dickens n'est donc pas seulement «l'explorateur infatigable de toutes les douleurs d'une interminable enfance ». Il entretient aussi, et peut-être surtout, un rapport particulier à la naissance. Ce n'est pas la mort, selon Isabelle Jan, qui est porteuse d'angoisses, mais bien les premiers instants de la vie.

L'hypothèse, aux accents psychanalytiques, contredit l'idée «qu'une littérature préfreudienne [ne] puisse être nourrie, gorgée même du traumatisme de la naissance ». C'est pourtant sur ce point précis, affirme Isabelle Jan, que se focalise l'art du romancier anglais. La naissance y est considérée, de manière inconsciente, comme un «processus de mise à mort ». La naissance et l'agonie ne sont plus que les « deux faces d'une même catastrophe ». Dans cette perspective, la Sara Gamp de Martin Chuzzlewit, « accoucheuse » atypique (qui donne son titre au sixième chapitre de l'ouvrage), figure «l'une des créations les plus hideuses et les plus terrifiantes de Dickens ». Le « sadisme » de 


\section{Livres en notes}

l'auteur s'exprime ici avec une force d'autant plus grande « qu'il se place dans le registre du comique ». Mais en même temps, avec ce personnage, Dickens virtualise et conjure l'effroi de la naissance, «en le renvoyant à l'imaginaire ». En guise de conclusion, le septième et dernier chapitre de l'essai d'Isabelle Jan revient néanmoins sur le désir «plus fort que tout », dans l'œuvre de Dickens, « de ne pas être au monde ». C'est dans la pension Todgers que l'on peut voir la métaphore la plus puissante de ce désir, «mais c'est l'œuvre tout entière », finit par affirmer l'auteur de l'essai, qui pose « la convergence absolue du naître et du mourir ».

L'analyse, menée avec brio et fermeté, ne manque pas d'éclat. On regrettera simplement que, dans le but louable de sortir Dickens des sentiers battus de l'écrivain pour enfants, Isabelle Jan ne revienne un peu paradoxalement sur un certain nombre d'avancées et de faits établis, au moins depuis la seconde moitié du $\mathrm{XX}^{\mathrm{e}}$ siècle, par la critique dickensienne, et ne diminue considérablement, dans les deux premiers tiers de son ouvrage, le sens et la portée des romans de l'auteur victorien. Divinités du tic définit le romancier comme un « observateur du jamais vu, de l'aléatoire, de l'inutile, créateur d'un univers qui, précisément, ne doit plus rien à l'observation, d'un univers hautement improbable » (chapitre 4). Pour stimulant qu'il soit, cet énoncé fait appel, en vue de sa validation, à des raccourcis et à de regrettables réductions d'une œuvre qu'il prétendait pourtant éclairer de nouveaux feux. Si elle a bien compris que Dickens n'était pas un réaliste, mais qu'il n'aspirait pas non plus " à l'idéalisme du bon victorien dans lequel on a souvent voulu le confiner ", si elle refuse, à raison, de lui appliquer le terme de «caricaturiste », Isabelle Jan n'en fait pas moins de Dickens le créateur de coquilles vides, qu'il remplirait à volonté, et sans souci de cohérence, de «tics », d' « outrance », « de divagations et de contorsions ».

Cette absurdité, pense Isabelle Jan, ne serait que le symptôme d'une vision plus tragique de l'existence; elle s'oppose au « raisonnement sociologique » à l'œuvre dans les romans de Balzac, et dont on ne retrouverait pas trace, en revanche, chez Dickens. Ce dernier, comme l'affirme autoritairement l'auteur de l'essai, ne serait pas intéressé quant à lui par l'ambiguïté. S'il atteint parfois le pathétique de la condition humaine, il ignorerait toutefois « la complexité du monde », et ne pourrait l'appréhender qu'avec naïveté. Reprenant une formule d'Henry James, qui qualifiait Dickens du «plus grand des romanciers superficiels » (the greatest of superficial novelists), Isabelle Jan tient ces propos quelque peu définitifs: «Dickens ne va pas au fond des choses et des êtres, mais tel n'est toutefois pas son objectif, si toutefois il en avait conçu un. Il ne se pose aucun défi, il n'est en quête de rien. C'est à lui plus qu'à quiconque que pourrait s'appliquer le mot fameux de Picasso: «Je ne cherche pas, je trouve ». » Elle annonçait d'emblée, dès son premier chapitre : «Il 
n'y a pas, chez [Dickens], de discours, il n'y a que des postures [...]. Pour fixer ses fantasmes, Dickens ne possédait qu'une technique encore embryonnaire ». Sylvère Monod, dans son ouvrage fameux sur Dickens romancier, a pourtant magistralement montré comment cette technique «embryonnaire » évolua en réalité dans le sens d'une maîtrise de plus en plus affirmée.

Les quelques pages consacrées plus loin à Sara Gamp tranchent curieusement avec cet énoncé, qui rend compte d'une perception un peu dépassée de l'œuvre du romancier victorien, et dont il est dommage qu'il serve à éclairer une préoccupation « inconsciente », celle de la naissance perçue comme traumatisme, et envisagée comme la clef de toute l'œuvre. Idée riche de suggestions mais qui, en l'état, ne peut que laisser le lecteur incrédule, tant elle est présentée de manière outrancière: l'enfance, chez Dickens, serait le sujet « d'un étrange et permanent holocauste ». Le rapprochement est saisissant, mais contestable, sinon légèrement tendancieux. On pourrait n'y voir qu'une regrettable figure de style s'il n'était repris par deux fois dans la suite de l'ouvrage: «L'accoucheuse », Sara Gamp, est présentée comme une " chienne, au même titre que les trop célèbres gardiennes du camp de Büchenwald », et les enfants de Nicolas Nickleby sont emprisonnées chez l'horrible Squeers « pour une raison assez semblable à celle qui, un siècle plus tard, présidera à l'ouverture des camps de la mort. En dépit des lettres dont ils sont affublés [...], ce sont des enfants sans nom. Ces sous-êtres portent la tare, indélébile, de ne pas avoir été désirés, accueillis et nommés ». C'est préférer, quitte à susciter la méfiance et à ne pas emporter l'adhésion, le clinquant d'une formule ambiguë à la rigueur d'une démonstration vraiment limpide. 